\title{
Integration of Weighted Terminological Concepts and Vague Knowledge in Ontologies for Decision Making
}

\author{
Nadine Mueller, Klemens Schnattinger \\ Baden-Wuerttemberg Cooperative State University, Loerrach, Germany \\ Email address: \\ muellnad@dhbw-loerrach.de (N. Mueller), schnattinger@dhbw-loerrach.de (K. Schnattinger)
}

\section{To cite this article:}

Nadine Mueller, Klemens Schnattinger. Integration of Weighted Terminological Concepts and Vague Knowledge in Ontologies for Decision Making. International Journal of Intelligent Information Systems. Vol. 8, No. 3, 2019, pp. 58-64. doi: 10.11648/j.ijiis.20190803.11

Received: May 15, 2019; Accepted: June 10, 2019; Published: July 30, 2019

\begin{abstract}
A well-known family of logics for managing structured knowledge is Description logics (DLs). They form the basis for a wide variety of ontology languages. Experience with the use of DLs in applications has, however, shown that their capabilities are insufficient for some domains. In particular, the decision-making process requires the assessment of two, possibly contradictory, influences on decision factors. First, there are items belonging to certain classes or fulfilling certain roles within complex logical constructs, but these memberships are to some extent vague. Secondly, individual preferences may change depending on the person who controls the decision-making process. Therefore, the challenge in building a decision making framework is to appropriately account for these variable influences by depicting and incorporating both aspects. This paper shows how these influences can be best modeled using a combination of fuzzy description logic and weighted description logic. Fuzzy logic is used to represent vagueness and ambiguity in ontologies, weighted description logic expresses individual preferences. In addition, the paper shows how to engineer an appropriate architecture for the suggested model.
\end{abstract}

Keywords: Ontology Learning, Weighted Description Logic, Fuzzy Logic, Decision Making

\section{Introduction}

Many decision-making processes require expert knowledge. Human experts can identify structural patterns of decision situations in order to model decision processes [1]. Cognitive-psychologically, decision making requires heuristics that ignore some of information in order to make decisions faster, more economical or more accurate. The ability to work with vague information is crucial in dealing with systems that are described by complex ontologies and consist of many instances [2]. Decision making and argumentation interact between processes that use logical thinking or heuristic reasoning. Therefore, one can argue that intuitive processes allow access to some form of logical reasoning. But it is also possible, that logic and rationality can be conceived as domains of explicit high-level forms of processing.

Description logics (DLs) offer a powerful tool to formally structure knowledge and support reasoning. When making decisions, it is often necessary to take both a set of formally structured requirements and individual preferences simultaneously into account. This requires an extension of the common knowledge bases, the so-called decision bases, which are initially based on the multi-attribute utility theory (MAUT) [3]. Since then, various approaches like the application of logic to decision and utility theoretical problems have emerged [4-6]. A common approach is to augment the framework with fuzzy logic as soon as ambiguity occurs [7, 8]. However, in cases where individual preferences encounter vague knowledge and assertions, neither decision bases nor fuzzy description logic alone can satisfy the paradigms. To close the gap, this paper provides a framework to model ambiguity and individual preferences at the same time. It combines the fuzzy description logic with the weighted description logic. To ease understanding, we first introduce the architecture used in this specific context. Then, to get a fine grasp of the combined framework of weighted description logic and fuzzy description logic, we will familiarize the reader with both separately. Initially, we establish the basics of the weighted description logic. We subsequently present the fuzzy description logic and focus on how it supports the modeling of ambiguous and vague 
knowledge. At the same time, the demarcation to probabilistic settings is highlighted. After combining these two approaches, the fuzzy decision base framework is introduced. Finally, we show how this framework can support the decision-making process within the respective architecture.

\section{Preliminaries}

The following sections present our architecture for opinion and consensus mining OMA, classical description logic and two extensions, the weighted description logic and fuzzy description logic.

\subsection{Opinion \& Consensus Mining Architecture OMA}

The original Opinion Mining Architecture (OMA) is part of a project of the same name. OMA was used for the first time for sentiment analysis of tweets for the financial sector [9]. In order to obtain an automated calculation of sentiment scores from texts, traditional approaches of natural language processing (such as POS tagging, parsing) and machine learning from texts (such as n-gram, syntactic/semantic features) were used for the pre-processing of the texts [10]. In addition, an extension of the description logic [11], the socalled weighted description logic [6], was used to automatically calculate the sentiment scores. The idea of separating the text processing task (filtering out relevant phrases) from the decision support task (evaluating extracted phrases) comes from the text understanding system SYNDIKATE [12] and its qualitative calculus [4].

In order to explain the extension of the OMA to include consensus mining and decision making, we first describe the essential components of the OMA. In Figure 1 we see (from top to bottom):

The TBox, which accommodates models of compliances, rules, judgements, etc.

The $A B o x$, which contains unweighted statements about the model of the TBox.

$U_{i}$ Boxes (on the right side) containing different preference models of experts.

From a technical point of view, the description logic represents the models of the TBox completely through terminological concepts, roles and is-a relations. The elements of the ABox are terminological assertions that enter into an instance-of-relationship with concepts of the TBox. At this point it should be noted that these assertions are created by the text-processing task from newspapers, social media, political programs, etc. (see the cloud on the left in Figure 1). The preference model of an expert $e_{i}$ is shown in the $U_{i} B o x$. A preference model consists of an a priori preference relation among attributes of concepts (see black circles in Figure 1). Each model represents the individual utility function of an expert $e_{i}$. From these a priori preference relations of an expert, a first a posteriori preference order can be derived for each expert choice (see the individual preference orders in Figure 1). Note that the preference model of each expert can be extracted a priori from the text processing task or entered directly by each expert. Next, the individual preference relations of each expert are used to build consensus or, in the case of only one expert, to directly retrieve the best possible choice respectively decision. The former is done by incomplete fuzzy preference relations for group decision making [13], which repeatedly adapts the preference relations of all experts until a satisfying consistent consensus is achieved. The theoretical basis of this approach is the use of an IOWA operator [14], which is also used in other combined methods [15].

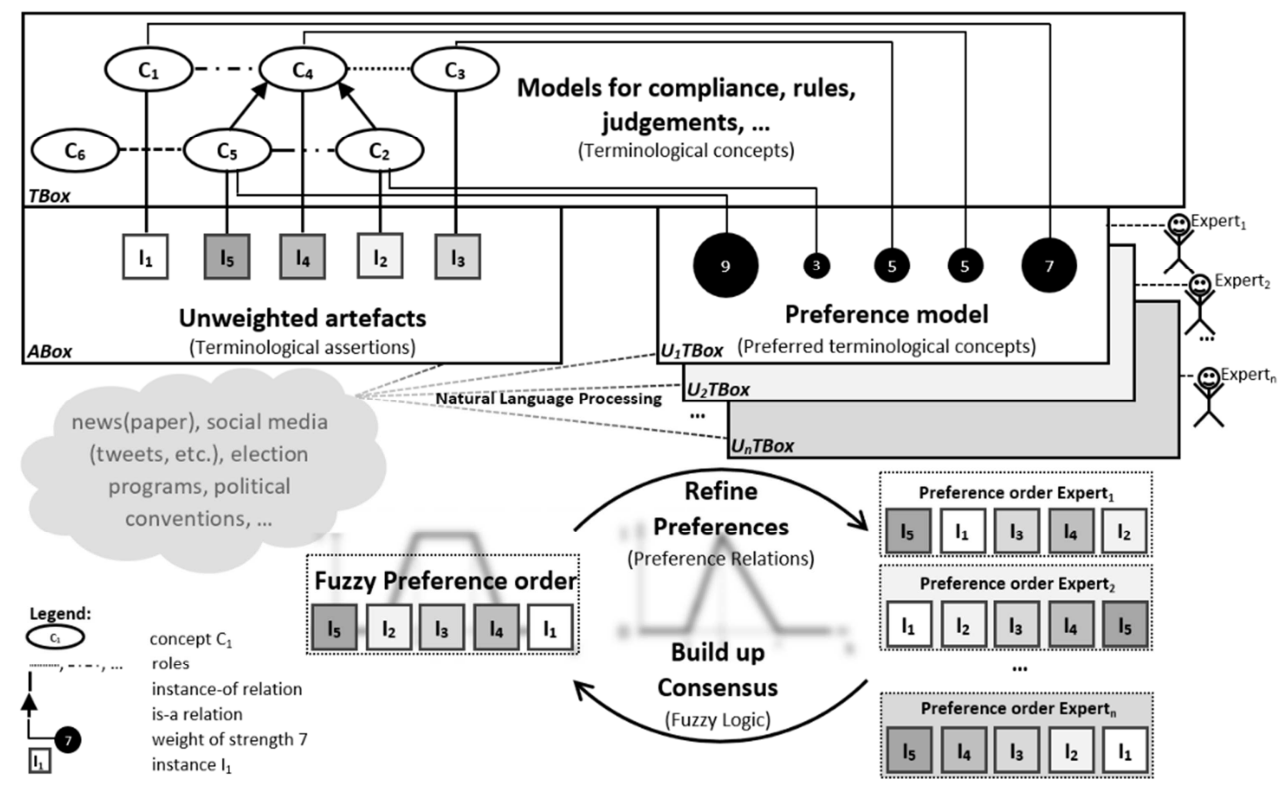

Figure 1. The Opinion \& Consensus Mining Architecture OMA.

\subsection{Description Logic}

Description logics (DLs) [11] are a family of logic-based knowledge representation formalisms. They can be used to represent and reason on the knowledge of an application 
domain. The basis of description logics is a common family of languages, known as description languages, that contain a set of constructors for ontologies consisting of concept (class) and role (property) descriptions.

A description language consists of an alphabet with unique concept names $\left(N_{C}\right)$, role names $\left(N_{R}\right)$ and individual (object) names $\left(N_{I}\right)$. In addition, so-called constructors are used to create concept and role descriptions. Depending on which constructors are allowed, there are many different description languages. Some of them form the basis for the ontology language of the semantic web [16].
The SROIQ Description Logic. A rather expressive description language is SROIQ-DL [17]. SROIQ-DL is compatible with OWL2, the current semantic web standard [18], making it the most reasonable description language within the above architecture. Below are some examples for the formal definition of the notions of SROIQ-roles and SROIQ-concepts as well as the underlying model-theoretic semantics. The interpretation is written as $I$, the domain as $\Delta^{I}$ and the interpretation function as $\cdot^{I}[17]$ :

Table 1. Example syntax and semantics of SROIQ-DL

\begin{tabular}{|c|c|c|}
\hline Constructor & Syntax & Semantics \\
\hline top & $\mathrm{T}$ & $\Delta^{j}$ \\
\hline bottom & $\perp$ & $\emptyset$ \\
\hline general negation & $\neg C$ & $\Delta^{\mathcal{J}} \backslash C^{\jmath}$ \\
\hline conjunction / disjunction & $C \sqcap D / C \sqcup D$ & $C^{\jmath} \cap D^{\jmath} / C^{\jmath} \cup D^{\jmath}$ \\
\hline exists restriction & $\exists R . C$ & $\left\{x \in \Delta^{\jmath} \mid \exists y .\langle x, y\rangle \in R^{\jmath} \wedge y \in C^{\jmath}\right\}$ \\
\hline value restriction & $\forall R . C$ & $\left\{x \in \Delta^{j} \mid \forall y .\langle x, y\rangle \in R^{j} \rightarrow y \in C^{j}\right\}$ \\
\hline at-most restriction & $\leq n R$ & $\left\{x \in \Delta^{j} \mid \#\left\{y \in \Delta^{j} \mid R^{\jmath}(x, y)\right\} \leq n\right\}$ \\
\hline at-least restriction & $\geq n R$ & $\left\{x \in \Delta^{\jmath} \mid \#\left\{y \in \Delta^{\jmath} \mid R^{\jmath}(x, y)\right\} \geq n\right\}$ \\
\hline concept definition / concept specialisation & $D \equiv C / D \sqsubseteq C$ & $D^{\jmath}=C^{\jmath} / D^{\jmath} \subseteq C^{\jmath}$ \\
\hline
\end{tabular}

In DLs, we distinguish between terminological knowledge (so-called $\mathcal{T}$ Box ) and assertional knowledge (so-called $\mathcal{A}$ Box). A $\mathcal{T}$ Box is a set of concept inclusions $C \sqsubseteq D$ and concept definitions $C \equiv D$. An $\mathcal{A}$ Box is a set of concept assertions $a: C$ as well as role assertions $(a, b): R$.

A so-called concrete domain $\mathcal{D}$ is defined as a pair $\left(\Delta^{\mathcal{D}}, \operatorname{pred}(\mathcal{D})\right) . \Delta^{\mathcal{D}}$ is the domain of $\mathcal{D}$ and pred $(\mathcal{D})$ is the set of predicate names of $\mathcal{D}$. The following assumptions were made: $\Delta^{\mathcal{J}} \cap \Delta^{\mathcal{D}}=\emptyset$ and for each $P \in \operatorname{pred}(\mathcal{D})$ with arity $\mathrm{n}$ there is $P^{\mathcal{D}} \subseteq\left(\Delta^{\mathcal{D}}\right)^{n}$. Functional roles are denoted by lowercase letters, for example with $r$ [11]. In description logics with concrete precise domains, $N_{R}$ consists of functional and ordinary roles. A role $r$ is functional if for each $(x, y) \in r$ and $(w, z) \in r$ it applies $x=w \Rightarrow y=z$. Functional roles are explained as partial functions of $\Delta^{\mathcal{J}}$ to $\Delta^{\mathcal{J}} \times \Delta^{\mathcal{D}}$. Within SROIQ all statements gathered about roles are captured in an $\mathcal{R}$ Box, which is not applied to our examples for reasons of clarity and compatibility with the basic description logic definitions [11].

Next, we extend an existing knowledge base [9] of a domain that will be used in the further course of the work. Its purpose is pure illustrative, so that reasoning and entailment is obvious. We will list explicit and implicit knowledge ("- $i k-")$ :

$$
\mathcal{T}=\{\text { Device } \sqsubseteq \mathrm{T}, \text { Equip } \sqsubseteq \mathrm{T}, \text { Device } \sqcap \text { Equip } \sqsubseteq \perp, \text { PoorEquip } \sqsubseteq \text { Equip, WellEquip } \sqsubseteq \text { Equip }
$$

PoorEquip $\sqcap$ WellEquip $\sqsubseteq \perp$, Device $\equiv \exists$ hasWeight. $>_{0 g} \sqcap \exists$ hasPrice. $>_{0 €} \sqcap \forall$ equipped.Equip

Tablet $\equiv$ Device $\sqcap$ ヨhasPrice. $>_{200 €}$, InexpensiveTablet $\equiv$ Tablet $\sqcap \exists$ hasPrice. $\leq_{500 €}$

ExpensiveTablet $\equiv$ Tablet $\sqcap$ GhasPrice. $\geq_{900 €}$, InexpensiveTablet $\equiv \neg$ ExpensiveTablet

ExpensiveTablet $\equiv \neg$ InexpensiveTablet, LightWeightTablet $\equiv$ Tablet $\sqcap \exists$ hasWeight. $\leq_{900} g$

Convertible $\sqsubseteq$ UpperclassTablet, UpperclassTablet $\equiv$ Tablet $\sqcap \forall$ equipped. WellEquip

LowerclassTablet $\equiv$ Tablet $\sqcap \forall$ equipped.PoorEquip

UpperclassTablet $\sqcap$ LowerclassTablet $\sqsubseteq \perp,-\mathrm{ik}-\}$

$\mathcal{A}=\left\{\right.$ tab $_{1}:$ Tablet $,\left(\right.$ tab $\left._{1}, 999 €\right):$ hasPrice $,\left(\right.$ tab $\left._{1}, 710 \mathrm{~g}\right):$ hasWeight

equipment ${ }_{1}$ :WellEquip, $\left(\right.$ (tab $_{1}$, equipment $\left._{1}\right)$ : $\forall$ equipped. WellEquip

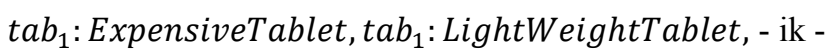

tab ${ }_{1}$ :UpperclassTablet, - ik-



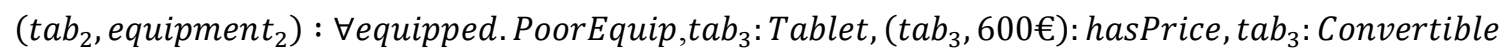




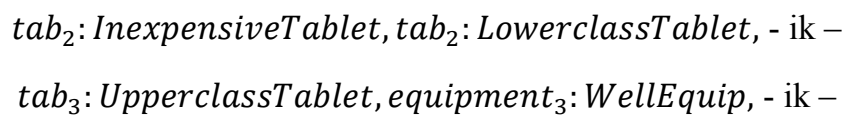

$\left.\left(\text { ta }_{3}, \text { equipment }\right)_{3}\right): \forall$ equipped.WellEquip - ik - $\}$

A considerable amount of knowledge is implicitly revealed in the terminological knowledge base. In this case a lot of knowledge about the domain is available, but does not provide support for a comprehensible decision. For this reason, the capabilities of the knowledge base are extended to include the ability to depict individual preferences.

\subsection{Weighted Description Logic}

The weighted description logic (WDL) can be regarded as a generic framework, the so-called decision base [6]. We use an a priori preference relation over attributes (the so-called ontological classes). Thereby, an a posteriori preference relation over choices (called ontological individuals) can be derived. Formally, a utility function $U$ over $\mathcal{X}$ (the set of attributes) is defined ( $U: \mathcal{X} \rightarrow \mathbb{R})$. Additionally, a utility function $u$, which is defined over choices and uses logical intentions, extends the utility function $U$ to the subset of choices [19]. Modelling attributes takes place in two steps:

1. Each attribute is modelled by a concept.

2. For every value of an attribute a new (sub) concept is introduced.

For instance, if equipped is an attribute to be modeled, it is simply represented by the Equipment concept (i.e. Equipment $\in \mathcal{X}$ ). An equipment can be regarded as a value, as if it was a concept of its own. If "well equipped" is a value of the attribute equipped, the attribute set $\mathcal{X}$ is simply extended by the concept WellEquip, as a sub-concept of Equipment. It should be noted that an axiom is introduced to guarantee disjointedness (e.g. PoorEquip ᄃ $\neg$ WellEquip) and that this procedure results in a binary term vector for $\mathcal{X}$, because an individual $c$ (as a choice) is either a member of a specific attribute of the concept set $\mathcal{X}$ or not.

A total preference relation (i.e. $\geqslant x$ ) over an ordered set of not necessarily atomic attributes $\mathcal{X}$ and a function $U: \mathcal{X} \rightarrow \mathbb{R}$ that represents $\geqslant$ (i.e., $U\left(X_{1}\right) \geq U\left(X_{2}\right)$ if $X_{1} \geqslant_{x} X_{2}$ for $\left.X_{1}, X_{2} \in \mathcal{X}\right)$ is given. Then the function $U$ assigns an a priori weight to each concept $X \in \mathcal{X}$. Therefore, one can say, that " $U$ makes the description logic weighted". The utility of a concept $X \in \mathcal{X}$ is denoted by $U(X)$. The following applies: The greater the utility of an attribute the more preferred it is.

As mentioned above, a choice is an individual $c \in N_{I}$. $\mathcal{C}$ denotes the finite set of choices. To determine a preference relation (a posteriori) over $\mathcal{C}$ (i.e. $\geqslant_{\mathcal{C}}$ ) which respects $\geqslant_{x}$, a utility function $u(c) \in \mathbb{R}$ is introduced. $u(c)$ indicates the utility of a choice $c$ relative to the attribute set $\mathcal{X}$. Also, a utility function $U$ over attributes as an aggregator is introduced. For simplicity, the symbol $\geqslant$ is used for both choices and attributes when it is evident from the context.

Within a consistent knowledge base $\mathcal{K}:=\langle\mathcal{T}, \mathcal{A}\rangle$, consisting of a $\mathcal{T}$ Box $\mathcal{T}$ and an $\mathcal{A B o x} \mathcal{A}$, the $\sigma$-utility is a particular $u$ and is defined as $u_{\sigma}(c):=\sum\{U(X) \mid X \in$
$\mathcal{X}$ and $\mathcal{K} \vDash \mathrm{c}: X\}$ and is referred to as the sigma utility of a choice $c \in \mathcal{C} . u_{\sigma}$ triggers a preference relation over $\mathcal{C}$ i.e., $u_{\sigma}\left(c_{1}\right) \geq u_{\sigma}\left(c_{2}\right)$ iff $c_{1} \geqslant c_{2}$. Each choice that is logically entailed e.g. $\mathcal{K} \vDash c$ : $X$ corresponds to a set of attributes. Due to the criterion additivity, each selection $c$ corresponds to a result.

Putting things (DL, $U$ and $u$ ) together it defines a generic $\mathcal{U}$ Box (so-called Utility Box) as a pair $\mathcal{U}:=\left(u_{\sigma}, U\right)$ where $U$ is a utility function over $\mathcal{X}$ and $u_{\sigma}$ is the utility function over $\mathcal{C}$. Furthermore, a decision base is defined as a triple $D=(\mathcal{K}, \mathcal{C}, \mathcal{U})$, where $\mathcal{C} \subseteq N_{I}$ is the set of choices and $\mathcal{U}=(u, U)$ is an $\mathcal{U}$ Box. Note: $\mathcal{K}$ provides assertional information about the choices and terminological information about the agent's ability to reason over choices.

Now we expand our tablet example with various utility boxes $\left(\mathcal{U}_{i}\right)$ and utility functions $\left(u_{i, \sigma}\right)$ from two experts:

For expert $1 U_{1}=\{($ InexpensiveTablet, 50), (UpperClassTablet, 40), (LightWeightTablet, 40$)\}$, $u_{1, \sigma}\left(t a b_{1}\right)=40+40=80, u_{1, \sigma}\left(t a b_{2}\right)=50$ and $u_{1, \sigma}\left(t a b_{3}\right)=40$. It follows that $t a b_{1}>t a b_{2}>t a b_{3}$

For the expert 2, however,

$\mathcal{U}_{2}=\{($ InexpensiveTablet, 60$)$,

(UpperClassTablet, 20), (LightWeightTablet, 10)\},

$u_{2, \sigma}\left(t a b_{1}\right)=20+10=30, u_{2, \sigma}\left(t a b_{2}\right)=60$ and $u_{2, \sigma}\left(t a b_{3}\right)=20$. It implies that $t a b_{2}>t a b_{1}>t a b_{3}$

Within this decision base, an expert with the utility box $\mathcal{U}_{1}$ would classify $t a b_{1}$ as first choice, whereas an expert with another utility box $\mathcal{U}_{2}$ would prefer $t a b_{2}$. At $t a b_{3}$ two different problems occur. One of them is that for this tablet a weight (in the sense of mass, not weighting of a concept according to WDL sense) is not known. Therefore, the reasoning fails if an instance check for this tablet is performed on the concept LightWeightTablet. For this reason, calculating the utility value is treated as if it were not an instance of LightWeightTablet. But the membership of this concept is unknown, and it cannot be reasoned that the instance does not belong to the concept LightWeightTablet. The other problem is that the tablet at a price of $600 €$ is neither inexpensive nor expensive (InexpensiveTablet resp. ExpensiveTablet). Although the price is well-known, the utility function treats this tablet just like expensive ones, which is not quite reasonable in this scenario. In order to eliminate this problem, the knowledge base is extended by fuzzy description logic and then combined with the decision base presented in the following chapters.

\subsection{Fuzzy Description Logic}

In order to deal with the ambiguity of the underlying domain, it is necessary to clarify where this uncertainty comes from. Either the uncertainty is due to a probabilistic cause or to vagueness. If the first situation occurs, then a statement is either "true" or "false" to a possibility (in the sense of likelihood), whereas in the second situation a 
statement is either "true" or "false" to some degree (in the sense of reaching a graded level) [20].

In the context of the choice of tablets mentioned above, the underlying ambiguity arises from vagueness. In order to model vague knowledge description logic is enriched with fuzzy logic, which enables the reflection of a degree of membership to a certain concept. A fuzzy set is defined by its characteristics, the so-called membership function [21].

Let $X$ be a non-empty set of individuals, then a class $A$ in $X$ is characterized by its membership function $f_{A}: X \rightarrow[0,1]$ and assigns to each $x \in X$ a real number within the interval $[0,1]$. This value represents the degree of which it belongs to $A$. The membership function defined for fuzzy sets has some essential properties which appear natural [22]:

$$
\begin{gathered}
\forall x \in X: A=\emptyset \text { iff } f_{A}(x)=0 \\
\forall x \in X: A^{\prime}=X \backslash A: f_{A^{\prime}}(x)=1-f_{A}(x) \\
\forall x \in X: A \subseteq B: f_{A}(x) \leq f_{B}(x) \\
\forall x \in X: A \cup B: f_{A \cup B}(x)=\max \left(f_{A}(x), f_{B}(x)\right) \\
\forall x \in X: A \cap B: f_{A \cap B}(x)=\min \left(f_{A}(x), f_{B}(x)\right)
\end{gathered}
$$

To enhance the possibilities of fuzzy sets, algebraic operations can also be defined. There are plenty of definitions e.g. Lukasiewicz logic, Gödel logic [23]. In this work the standard fuzzy logic (SFL) is used, but all others can also be applied. Some definitions can be found in table 2 .

This toolset of fuzzy set definitions and algebraic operators can now be applied to description logics to reflect ambiguity and vagueness in knowledge bases. For example, an individual that is only an instance of a concept to a certain degree can be modelled suitably.

Table 2. Definitions of algebraic operations,

\begin{tabular}{ll}
\hline Algebraic operator & SFL \\
\hline$a \otimes b / a \oplus b$ & $\min (a, b) / \max (a, b)$ \\
$a \Rightarrow b / \Theta a$ & $\max (1-a, b) / 1-a$ \\
\hline
\end{tabular}

To formally quote this fuzziness of description logic axioms, we use the syntax presented in Straccia [7]. The conceptional syntax of fuzzy description logics is the same as for the description logics defined above (see chapter 2.2). The semantics, however, reflects the fuzzy logic. Therefore, a fuzzy interpretation is a pair $\mathcal{J}=\left(\Delta^{\mathcal{J}},{ }^{\mathcal{J}}\right)$ consisting of a non- empty set called domain and a fuzzy interpretation function. This function maps individuals as usual and concepts into membership functions $\Delta^{j} \rightarrow[0,1]$. Accordingly, the roles are mapped to $\Delta^{\mathcal{J}} \times \Delta^{\mathcal{J}} \rightarrow[0,1]$. Consequently $\mathcal{C}^{\mathcal{J}}$ is the membership function of the fuzzy set $C$. Hence, a concept is interpreted as fuzzy set.

Example

A specific tablet is an instance of the concept Convertible only to a certain degree depending on its features. We therefore extend the description logic and allow this degree to be captured as a fuzzy value and write $\left\langle t a b_{3}:\right.$ Convertable, 0.8$\rangle$. This means that $t a b_{3}$ is at least one instance of the concept Convertable with the degree of 0.8. Analogously, Convertable ${ }^{J}\left(\mathrm{tab}_{3}\right)$ returns the minimal degree that $t a b_{3}$ is a convertible tablet under the interpretation $\mathcal{J}$.

The properties of fuzzy sets and algebraic operators are now applied to interpretations of SROIQ and lead to the following example rules for all $d \in \Delta^{\mathcal{J}}$ [24]:

Table 3. Fuzzy semantics (non-exhaustive).

\begin{tabular}{ll}
\hline Syntax & Semantics \\
\hline$C \sqcap D$ & $(C \sqcap D)^{\jmath}(d)=\min \left\{C^{\jmath}(d), D^{\jmath}(d)\right\}$ \\
$\neg C$ & $(\neg C)^{\jmath}(d)=1-C^{\jmath}(d)$ \\
$C \sqsubseteq D$ & $(C \sqsubseteq D)^{\jmath}=\inf _{d \in \Delta^{\jmath}} C^{\jmath}(d) \Rightarrow D^{\jmath}(d)$ \\
$\exists R . C$ & $(\exists R \cdot C)^{\jmath}(a)=\sup _{b \in \Delta^{\jmath}}\left\{\min \left(R^{\jmath}(a, b), C^{\jmath}(b)\right)\right\}$ \\
\hline
\end{tabular}

Example

Let $\mathcal{K}$ be the knowledge base above, but now the concept LightWeightTablet is no longer strictly defined according to classic DL, but intuitively with the help of fuzzy DL. Within the $\mathcal{T}$ Box, the row $\exists$ hasWeight. $\leq_{900 g}$ 드 LightWeightTablet will be replaced by the following two constructs:

$$
\begin{gathered}
\left\langle\left(\exists \text { hasWeight }_{\cdot \geq 900 g} \sqcap \exists \text { hasWeight }_{\cdot \leq 1100 g}\right)\right. \\
\text { LightWeightTablet }, 0.6\rangle
\end{gathered}
$$

\section{$\left\langle\exists\right.$ hasWeight $_{. \leq 900 \mathrm{~g}} \sqsubseteq$ LightWeightTablet, 1$\rangle$}

indicating that every tablet with a weight of less than $1100 \mathrm{~g}$ should still be considered a light tablet to a certain degree (here 0.6). For $t a b_{3}$ the exact weight is not known, but the related information varies between $900 \mathrm{~g}$ and $1100 \mathrm{~g}$ with strong tendencies to the upper threshold. Therefore the $\mathcal{A}$ Box is adjusted accordingly:

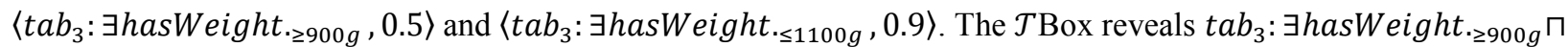

$$
\begin{aligned}
& \text { tab }_{3}: \exists \text { hasWeight. }{ }_{: 1100 g}=\min \left\{\text { tab }_{3}: \exists \text { hasWeight }{ }_{: \geq 900 \mathrm{~g}}, \text { tab }_{3}: \exists \text { hasWeight }_{{ }_{\leq 1100 g}}\right\}=\min \{0.5,0.9\}=0.5
\end{aligned}
$$

and $t a b_{3}$ is therefore a LightWeightTablet with the minimal degree of $\max \{1-0.5,0.6\}=0.6$.

\section{Weighted Fuzzy Description Logic}

For the weighted fuzzy description logic, the background knowledge base $\mathcal{K}=(\mathcal{T}, \mathcal{A})$ will also be able to capture vague knowledge and assertions formally referred to as $\mathcal{K} \approx(\mathcal{T}, \mathcal{A})$. This knowledge base is then extended by the set of choices $\mathcal{C}$ and the utility box $\mathcal{U}$ steering the decision making. The advantage of this framework is that these weights can be articulated independently and do not need to be compared against each other like in a more straightforward approach [25].

Definition

A triple $\mathcal{D} \approx(\mathcal{K}, \mathcal{C}, \mathcal{U})$, where $\mathcal{K}$ is a fuzzy knowledge base, $\mathcal{C}$ a set of choices and $\mathcal{U}$ is a utility box called a fuzzy decision base.

Note: Entities of the $\mathcal{U}$ Boxes are concepts that are relevant to the decision-making process, including their specific 
individual weights. After the reasoning for each of the existing choices, the instance check completes and reveals whether a choice belongs to a specific concept or not. Suppose a choice $c: U$ belongs to a concept, this could be vague even within the fuzzy description logics. Hence, it leads to constructs like $\langle c: U, n\rangle$ with $n \in[0,1]$.

Definition

Let $\langle c: U, n\rangle$ be a fuzzy assertion and $(U, w)$ a weighted attribute, then a fuzzy utility value of $c$ respective to $U$ is $u_{f \sim \sigma}(c: U) \stackrel{\text { def }}{=} w \cdot n$

If the assertion is not fuzzy, then $n$ is simply set to 1 . If the instance $c$ belongs to the complement of $U$ with a membership degree of 1 , then the fuzzy utility value for $c$ on this attribute is 0 (as $n$ is then 0 ).

Example

In case for $t a b_{3}$, the calculated respective reasoned membership degree for a lightweight tablet is 0.6 , formally written $\left\langle\right.$ tab $_{3}$ :LightWeightTable, 0.6〉 and expert $U_{1}$ defines for this attribute a weight of 40 , formally written as (LightWeightTablet, 40$)$, then $u_{f \sim \sigma}\left(t a b_{3}\right)=24$. The individual weight is a bit more than half of the initially defined one, since the degree of membership of this tablet is only 0.6. As the utility function is additive, the utility measure for a choice is the sum of all relevant attributes.

Definition

Let $\mathcal{D} \approx(\mathcal{K}, \mathcal{C}, \mathcal{U})$ be a fuzzy decision base with a utility box $\mathcal{U}$ of the cardinality $k=|\mathcal{U}|$, then the $\mathcal{U}$ Box fuzzy utility value of $c$ is $u_{f \sim u, \sigma} \stackrel{\text { def }}{=} \sum_{i=1}^{k} u_{f \sim \sigma}\left(c: U_{i}\right)$.

By calculating the $\mathcal{U}$ Box fuzzy utility values of each choice $c$ a total ordering of the $\operatorname{set} \mathcal{C}$ is naturally given. The ideal solution is therefore the choice with the highest fuzzy utility value relative to the $\mathcal{U}$ Box.

Definition

Let $\mathcal{D} \approx(\mathcal{K}, \mathcal{C}, \mathcal{U})$ be a fuzzy decision base with a utility box $\mathcal{U}$ of the cardinality $k=|\mathcal{U}|$, then the ideal fuzzy choice is $c_{f \sim s} \stackrel{\text { def }}{=} \arg \max _{c \in \mathcal{C}}\left(\sum_{i=1}^{k} u_{f \sim \sigma}\left(c: U_{i}\right)\right)$.

Example

For an expert with the utility box $U_{1}$ the summarized utility value for $t a b_{3}$ is $\sum_{i=1}^{k} u_{f \sim \sigma}\left(c: U_{i}\right)=24+40=64$. The ranking of choices for this expert changes to $t a b_{1}>$ $t a b_{3}>t a b_{2}$, which means that $t a b_{3}$ is preferred to $t a b_{2}$. In this scenario, the problem remains that the price of the tablet is neither expensive nor inexpensive, but unknown. Therefore, it is indispensable to design a consistent respective complete fuzzy decision base by ensuring that each attribute listed in the $\mathcal{U}$ Box is correct and decidable in the knowledge base.

Definition

A fuzzy decision base $\mathcal{D} \approx(\mathcal{K}, \mathcal{C}, \mathcal{U})$ is called complete if for every relevant attribute out of the $\mathcal{U}$ Box $U \in \mathcal{U}$ a fuzzy value for every $c \in \mathcal{C}$ is deducible: $\forall U \in \mathcal{U}, \forall c \in \mathcal{C} \exists n \in$ $[0,1]:\langle c: U, n\rangle$. Thus the fundamentals are defined to make a reasonable decision in the above scenario.

To complete the fuzzy decision base of the example above, $t a b_{3}$ requires a fuzzy value for the attribute "InexpensiveTablet" and tab $b_{2}$ for "LightWeightTablet". Therefore the $\mathcal{T}$ Box is extended to reveal fuzzy values also for weights above $1100 \mathrm{~g}$ and prices in between $500 €$ and
$900 €$. The following expressions are added to the $\mathcal{T}$ Box:

$$
\begin{aligned}
& \left\langle\neg \exists_{\text {hasWeight }} \leq 1100 \mathrm{~g} \subseteq \text { LightWeightTablet, } 0\right\rangle \\
& \left\langle\left(\exists \text { hasPrice }{ }_{>}>500 € \Pi \exists \text { hasPrice }{ }_{<990 €}\right)\right. \\
& \text { 들 }
\end{aligned}
$$

$$
\begin{gathered}
\left\langle\left(\exists \text { hasPrice } ._{>500 €} \Pi \exists \text { HasPrice }_{.<900 €}\right)\right. \\
\text { ExpensiveTablet }, 0.5\rangle
\end{gathered}
$$

The first line indicates that tablets with a weight above $1100 \mathrm{~g}$ do not belong to the concept LightWeightTablet at all. The fuzzy values of the second and third line represent the membership degrees of those tablets which have a price within this interval and is set manually to 0.5 as arithmetic mean between the two categorizations inexpensive and expensive. Thus, $t a b_{3}$ is a member of the concept Inexpensive with a degree of 0.5 and a member of Expensive with the same degree.

With standard fuzzy logic the fuzzy value of a concept's complement is: $(\neg C)^{\mathcal{J}}(d)=1-C^{\mathcal{J}}(d)$, which entails the following implicit knowledge:

\section{$\left\langle\right.$ InexpensiveTablet $\Pi \exists$ hasPrice $\left._{\geq 900 €}, 0\right\rangle$}

For example, if $t a b_{1}$ has the price of $999 €$, then the following applies:

$$
\begin{gathered}
\left\langle\text { ta }_{1}: \text { ExpensiveTablet }, 1\right\rangle \text { and } \\
\left\langle\text { tab }_{1}: \neg \text { ExpensiveTablet }, 0\right\rangle
\end{gathered}
$$

By means of this complete decision base the above decision can be derived properly. The utility values for $t a b_{3}$ within $\mathcal{U}_{1}$ and $\mathcal{U}_{2}$ are:

$$
\begin{aligned}
& u_{f \sim 1, \sigma}\left(\operatorname{tab}_{3}\right)=0.5 \cdot 50+40+0.6 \cdot 40=89 \\
& u_{f \sim 2, \sigma}\left(\operatorname{tab}_{3}\right)=0.5 \cdot 60+20+0.6 \cdot 10=56
\end{aligned}
$$

For an expert with the utility box $U_{1} t a b_{3}$ is his or her first choice, while the other expert still chooses $t a b_{2}$.

\section{Conclusion}

By extending the fuzzy logic by a decision base, a model is defined, that represents reality much better. Because of the uncertainty around $t a b_{3}$ a first calculation in a conventional decision base revealed a distorted result. Incorporating the vague knowledge existing in this domain, the expert would have chosen $t a b_{3}$ instead of $t a b_{1}$. The strength of this framework is that vague assertions are properly deliberated together with individual preferences.

It becomes obvious how the weighting influences the decision. As the first utility box has almost balanced weights, the second one has a strong tendency towards inexpensive tablets. Using this $\mathcal{U}$ Box, the first choice is still $t a b_{2}$. But the second choice is now $t a b_{3}$ and not $t a b_{1}$. Both tablets were initially not included in the concept InexpensiveTablet, but with the help of fuzzy logic the strong preference for inexpensive tablets causes $t a b_{3} t a b_{1}$ to pass.

Summarized, complete fuzzy decision bases offer a strong opportunity to model real world situations which need to 
respect ambiguity and individual preferences while supporting a comprehensible decision-making process. Further research needs to reveal supporting algorithms to detect and locate incompleteness to support the creation of complete fuzzy decision bases. Overall, creating these underlying ontologies is time-consuming and a non-trivial, manual process. To facilitate this, new approaches with deep learning algorithms have arisen [26]. The use of such techniques is another milestone on the way to a fully automated decision-making process.

\section{References}

[1] Jung, J., Lee, H., Choi, K.: Contextualized Recommendation Based on Reality Mining from Mobile Subscribers. Cybernetics and Systems. 40 (2), 160-175 (2009).

[2] Gigerenzer, G., Gaissmaier, W.: Heuristic Decision Making. Annual Review of Psychology 62, 451-482 (2011).

[3] Keeney, R., Raiffa, H.: Decisions with Multiple Objectives: Preferences and Value Trade-offs. Cambridge University Press, First published in 1976 by John Wiley \& Sons, Inc. (1993).

[4] Schnattinger, K., Hahn, U.: Quality-Based Learning. ECAI'98: Proc. 13th Biennial European Conference on Artificial Intelligence, Brighton, UK, 160-164 (1998).

[5] Lafage, C., Lang, J.: Logical Representation of Preferences for Group Decision Making. KR'00: Proc. 7th Conference on Principles of Knowledge Representation and Reasoning, 457468 (2000).

[6] Acar, E., Fink, M., Meilicke, C., Thome, C., Stuckenschmidt, H.: Multi-attribute Decision Making with Weighted Description Logics. IFCoLog: Journal of Logics and its Applications 4, 1973-1995 (2017).

[7] Straccia, U.: Reasoning within Fuzzy Description Logics. Journal of Artificial Intelligence Research (14), 137-166 (2001).

[8] Schnattinger, K., Mueller, N., Walterscheid, H.: Consensus Mining - A Guided Group Decision Process for the German Coalition Negotiations. FLAIRS'18: Proc. 31st Florida Artificial Intelligence Research Symposium, Melbourne, USA, 205-208 (2018).

[9] Schnattinger, K., Walterscheid, H.: Opinion Mining Meets Decision Making: Towards Opinion Engineering. IC3K'17: Proc. 9th International Joint Conference on Knowledge Discovery, Knowledge Engineering and Knowledge Management, 334-341 (2017).

[10] Sun, S., Luo, C., Chen, J.: A Review of Natural Language Processing Techniques for Opinion Mining Systems. Information Fusion 36, 10-25 (2017).

[11] Baader, F., McGuinness, D., Narci, D., Patel-Schneider, P.: The Description Logic Handbook: Theory, Implementation, and Applications. Cambridge University Press (2003).
[12] Hahn, U., Schnattinger, K.: Towards Text Knowledge Engineering. AAAI'98: Proc. 15th National Conference on Artificial Intelligence, 524-531 (1998).

[13] Herrera-Viedma, E., Alonso, S., Chiclana, F., Herrera, F.: A Consensus Model for Group Decision Making Incomplete Fuzzy Preference Relations. IEEE Transactions on Fuzzy Sytems 15 (5), 863-877 (2007).

[14] Yager, R. R., Filev, D. P.: Operations for Granular Computing: Mixing Words and Numbers. IEEE International Conference on Fuzzy Systems 2 (1), 123-128 (1998).

[15] Mueller, N., Schnattinger, K., Walterscheid, H.: Combining Weighted Description Logic with Fuzzy Logic. IPMU'18: Proc. 17th International Conference on Information Processing and Management of Uncertainty in KnowledgeBased Systems, 124-136 (2018).

[16] Horrocks, I., Patel-Schneider, P. F., McGuinness, D. L., Welty, C. A.: OWL: a Description Logic Based Ontology Language for the Semantic Web. In: Description Logic Handbook, 458486. Cambridge University Press, Cambridge (2007).

[17] Horrocks, I., Sattler, U., Kutz, O.: The even more irresistible SROIQ. KR'06: Proc. 10th Conference on Principles of Knowledge Representation and Reasoning, 57-67 (2006).

[18] Motik, B., Patel-Schneider, P. F., Cuenca Grau, B.: OWL 2 Web Ontology Language: Direct Semantics (Second Edition). (Accessed April 2018) Available at: http://www.w3.org/TR/2012/REC-owl2-direct-semantics20121211/.

[19] Fishburn, P.: Utility Theory for Decision Making. R. E. Krieger Publications \& Co, Huntington, N. Y (1979).

[20] Dubois, D., Prade, H.: Possibility theory, probability theory and multiple-valued. Annals of Mathematics and Artificial Intelligence 32 (1-4), 35-66 (2001).

[21] Zadeh, L. A.: A Computational Approach to Fuzzy Quantifiers in Natural Languages. Computers \& Mathematics with Applications 9 (1), 149-184 (1983).

[22] Hájek, P.: Metamathematics of Fuzzy Logic. Springer, Dordrecht; Netherlans (1998).

[23] Straccia, U.: All About Fuzzy Description Logics and Applications. In Faber, W., Paschke, A., eds.: Reasoning Web. Web Logic Rules, Cham, 1-31 (2015).

[24] Stoilos, G., et. al.: Reasoning with Very Expressive Fuzzy Description Logics. Journal of Artificial Intelligence Research (30), 273-320 (2007).

[25] Yager, R., Basson, D.: Decision Making with Fuzzy Sets. Fuzzy Sets and Systems, 87-95 (1978).

[26] Petrucci G., Ghidini C., Rospocher M.: Ontology Learning in the Deep. In: Blomqvist E., Ciancarini P., Poggi F., Vitali F. (eds) Knowledge Engineering and Knowledge Management. EKAW 2016. Lecture Notes in Computer Science, Springer, 480-495 (2016). 